\title{
Double vision in Parkinson's Disease: a systematic review
}

\author{
Katarzyna Śmiłowska ${ }^{1}$, Bogumił Wowra ${ }^{2}$, Jarosław Sławek ${ }^{3,4}$ \\ Department of Neurology, Regional Specialist Hospital of St. Barbara in Sosnowiec, Poland \\ ${ }^{2}$ Clinical Department of Ophthalmology, School of Medicine with the Division of Dentistry in Zabrze, Medical University of Silesia, \\ Katowice, Poland \\ ${ }^{3}$ Department of Neurological and Psychiatric Nursing, Medical University of Gdansk, Poland \\ ${ }^{4}$ Department of Neurology, St. Adalbert Hospital, Gdansk, Poland
}

\begin{abstract}
Introduction. The prevalence of diplopia in Parkinson's disease (PD) ranges from 10\% to $30 \%$. Diplopia is usually limited to specific situations such as reading and looking around, however, it can also be constant. The aim of this review, supplemented by two case studies, was to summarise current knowledge on the frequency, possible causes, differential diagnosis, pathophysiology and treatment approaches to double vision in PD patients.

Methodology. An electronic search (PubMed, Scopus, Embase and Web of Science) to identify studies regarding diplopia in PD patients was performed in May 2020. The following search terms were used: Parkinson's Disease and diplopia.

Results. Five articles are included in this literature review. In the literature, the number of included patients with PD who presented with symptoms of diplopia varied from a single case report to 88 patients. Diplopia was usually intermittent and binocular. The most commonly reported type of diplopia was single object diplopia, and the second most common was complete diplopia. Risk factors contributing to developing diplopia were: older age, disease duration, disease severity, cognitive decline, presence of visual hallucinations, and higher levodopa equivalent dose. Results also showed that diplopia had a significant impact on quality of life. Possible comorbidities of diplopia include myasthenia gravis and vascular disease.

Conclusion. Diplopia is relatively common in PD, but still understudied. The research shows that it has a clear impact on health-related quality of life and patient safety. The acute onset of diplopia should always result in tests to exclude other diseases. Tailored interventions should be developed, leading to improved patient safety, quality of life, and quality of care.
\end{abstract}

Key words: Parkinson's Disease, diplopia, double vison, myasthenia gravis

(Neurol Neurochir Pol 2020; 54 (6): 502-507)

\section{Introduction}

Parkinson's Disease (PD) is a neurodegenerative disorder presenting with primary motor symptoms (bradykinesia, tremor, rigidity and postural impairment), as well as a wide spectrum of non-motor problems [1,2]. Neuro-ophthalmological symptoms are common in PD but still understudied [3]. These symptoms may reflect ocular or vision-related (affecting visual pathway) impairment. Visual impairment in PD can be linked to retinal dopamine depletion or to decreased dopamine innervations of the visual cortex $[4,5]$. Dopamine, as a neurotransmitter, plays an important role in light adaptation, oculomotor control, contrast sensitivity, colour vision, and visuospatial construction and spatial working memory $[6,7]$. Thus, dopamine deficiency is linked to various visual disturbances in PD, including eyelid apraxia and blepharospasm, dry eyes syndrome, diplopia, primary open angle glaucoma, decreased colour and contrast vision, visuospatial impairment and hallucinations $[1,6]$. These symptoms have also been linked with disease severity, increased risk of falls and dementia $[3,8]$. Eye movement abnormalities can be used to distinguishing between PD and atypical parkinsonism. Specifically progressive supranuclear palsy is characterised by vertical smooth pursuit impairment followed by voluntary

Address for correspondence: Katarzyna Śmiłowska, Department of Neurology, Regional Specialist Hospital of St. Barbara in Sosnowiec, Plac Medyków 1 Str., 41-200 Sosnowiec, Poland, email: kasia.smilowska@gmail.com 
gaze palsy, with lid-opening apraxia and blepharospasm, but usually with no diplopia reported $[9,10]$. Additionally, visual problems can result in decreased quality of life for people with PD, limiting social activities, reading and other hobbies. Diplopia is now considered a part of the clinical spectrum of PD [11]; however, it requires a thorough diagnostic process in order to exclude other potential causes requiring a different therapeutic approaches.

\section{Clinical vignettes}

\section{Patient 1}

The first patient was a 66-year-old Caucasian woman with a 5-year history of PD diagnosed according to the United Kingdom Parkinson's Disease Society Brain Bank Diagnostic Criteria. Initially, the patient presented with hypomimia, a rest tremor of the left hand, rigidity and bradykinesia that was more pronounced on the left side. At that time, the patient was treated with piribedil and levodopa/benserazide controlled release (levodopa equivalent dose [LED] of $570 \mathrm{mg}$ ). Five years after PD diagnosis, the patient developed diplopia. The symptoms varied during the day, and diplopia was considered to be related to fatigue. The impairment of neuromuscular transmission was confirmed by the presence of anti-acetylcholine receptor antibodies as well as single-fibre electromyography of orbicularis oculi and extensor digitorum communis muscles, which showed an increased jitter value, increased pairs with abnormal jitter, and the presence of blocks. Chest computed tomography excluded the presence of thymoma. After the diagnosis of myasthenia gravis (MG), treatment with pyridostigmine $180 \mathrm{mg}$ per day was started, and the patient showed clinical improvement. Within three months of treatment initiation, the patient developed dysphagia, dyspnoea and dysarthria which progressed into a myasthenic crisis. The patient was mechanically ventilated and treated with intravenous immunoglobulins. During rehabilitation, she experienced a fall sustaining a hip fracture, and within a month after surgery developed a second myasthenic crisis and died at the age of 68 .

\section{Patient 2}

The second patient was a 57-year-old, right-handed Caucasian man with a seven-year history of PD diagnosed according to the UK Brain Bank Diagnostic Criteria. Her initial symptoms included hypomimia, bradyphrenia, a rest tremor of 4-6 Hz on the right, bradykinesia and cog-wheel rigidity that was more pronounced on the right side. Six years after starting levodopa treatment, the patient began to experience dyskinesias. The patient was treated with levodopa / benserazide (LED of $625 \mathrm{mg}$.). In the seventh year of disease, the patient developed horizontal diplopia consistent with motor 'off' periods. Diplopia occurred mostly while reading. The patient underwent an ophthalmological examination and was diagnosed with convergence insufficiency present only during the motor 'off' periods. Uptitration of levodopa improved her symptoms.
The aim of our review, supplemented by these two case reports, is to review current knowledge regarding possible causes, differential diagnosis, pathophysiology and treatment approach to double vision in PD, with a focus on diplopia in the course of PD and possible co-morbidities like MG. However, vascular causes of diplopia (e.g., ischemic third, fourth or sixth nerve palsy) are beyond the scope of this review but should always be considered during diagnosis.

\section{Methodology}

The electronic search to identify and capture literature regarding diplopia in PD patients was performed in May 2020. The following medical subject headings (MeSH) search terms were used: 'Parkinson's Disease' [MeSH] and 'diplopia' [MeSH]. In addition, reference lists of identified articles were screened for other potentially relevant papers.

We searched the PubMed, Scopus, Embase and Web of Science databases. No year restrictions were applied. To make the search as comprehensive as possible, we used the Boolean operators "AND" to link the search terms. Inclusion criteria were English-language, full-text articles in peer-reviewed academic/scientific journals that were available electronically. Studies on animal models and conference abstracts were excluded.

\section{Results}

In total, five articles were retrieved from the databases [12-16]. Two studies were observational cross-sectional studies [12,16] and three were case reports [13-15] including two case series $[13,14]$. Detailed information on the analysed articles is presented in Table 1.

Three studies compared patients with both PD and diplopia to healthy controls $[12,16]$, two examined PD patients with diplopia and PD patients both with and without diplopia $[12,13]$; and one reported on PD patients with diplopia, PD patients without diplopia, and healthy controls [12]. The number of included PD patients presenting symptoms of diplopia varied from a single case report [15] to 88 [16]. The age of the patients with diplopia ranged from 67 [14] to 72 [12] years. Disease duration ranged from six [12] to 14 [14] years. Modified Hoehn and Yahr stage, when given, ranged from $2.5[14]$ to $3[12,13]$.

The presence of binocular, intermittent, diplopia ranged from $60 \%(n=27)$ [13] to $100 \%(n=25)$ [12] of PD patients.

Visser et al. [12] reported the prevalence of daily and weekly binocular diplopia in $40 \%(n=10)$ and $60 \%(n=9)$ of PD patients, respectively.

The most commonly reported type of diplopia was single object diplopia, followed by complete diplopia (diplopia of the entire visual field) [12-14]. In Visser et al.s [12] study, only $18 \%(n=5)$ of patients had episodes of both selective and complete diplopia. 
Table 1. Summary of the included studies on diplopia in Parkinson's Disease

\begin{tabular}{|c|c|c|c|c|c|c|c|}
\hline $\begin{array}{l}\text { Author } \\
\text { [year] }\end{array}$ & $\begin{array}{l}\text { Number } \\
\text { of patients }\end{array}$ & Age & Male $n$ & $\begin{array}{l}\text { Disease } \\
\text { duration }\end{array}$ & $\begin{array}{l}\text { Hoehn } \\
\text { and Yahr }\end{array}$ & Type of study & $\begin{array}{l}\text { MDS-UPDRS } \\
\text { III }\end{array}$ \\
\hline \multirow[t]{3}{*}{$\begin{array}{l}\text { Visser et al. } \\
\text { [2019] [10] }\end{array}$} & $\begin{array}{l}\text { PD with diplopia1 } \\
\qquad n=25\end{array}$ & $72(9)$ & 18 & $6(5)$ & $3(1)$ & \multirow[t]{3}{*}{$\begin{array}{l}\text { Observational cross- } \\
\text {-sectional design }\end{array}$} & $24(9)$ \\
\hline & $\begin{array}{l}\text { PD without diplopia } \\
\qquad n=16\end{array}$ & $73(8)$ & 10 & $6(5)$ & $2(0)$ & & $19(10)$ \\
\hline & $\mathrm{HCn}=23$ & $70(8)$ & 12 & NA & NA & & NA \\
\hline \multirow[t]{2}{*}{$\begin{array}{l}\text { Schindlbeck et al. } \\
\text { [2017] [11] }\end{array}$} & $\begin{array}{l}\text { PD with diplopia } \\
\qquad n=37\end{array}$ & $70.5 \pm 9.3$ & 24 & $10.1 \pm 5.5$ & $3.0 \pm 0.9$ & \multirow[t]{2}{*}{ Case series } & $36.4 \pm 12.1$ \\
\hline & $\begin{array}{l}\text { PD without diplopia } \\
\qquad n=88\end{array}$ & $73.3 \pm 8.0$ & 41 & $6.4 \pm 7.3$ & $2.5 \pm 0.7$ & & $31.5 \pm 10.3$ \\
\hline \multirow[t]{2}{*}{$\begin{array}{l}\text { Urwyler et al. } \\
\text { [2014] [14] }\end{array}$} & $\begin{array}{c}P D \\
n=88\end{array}$ & $72.3 \pm 9.1$ & 47 & $8.6 \_5.6$ & ND & \multirow[t]{2}{*}{ Cross-sectional study } & $22.5 \pm 10.6$ \\
\hline & $\begin{array}{c}\mathrm{HC} \\
\mathrm{n}=90\end{array}$ & $73.5 \pm 8.4$ & 38 & NA & NA & & NA \\
\hline $\begin{array}{l}\text { Nebe et al. [2007] } \\
\text { [12] }\end{array}$ & $\begin{array}{c}P D \\
n=14\end{array}$ & 67 years & 6 & 14 & $2,5-4$ & Case series & ND \\
\hline $\begin{array}{l}\text { Racette et al. } \\
\text { [1999] [13] }\end{array}$ & $\begin{array}{c}P D \\
n=1\end{array}$ & 47 years & 1 & 10 & ND & Case report & ND \\
\hline
\end{tabular}

'diplopia defined as daily or weekly complaints of diplopia; ND — no data, NA — not applicable, HC — healthy controls, MDS-UPDRS — Movement Disorders Society-Unified Parkinson's Disease Rating Scale, PD - Parkinson's Disease

Urwyler et al. [16] found that the prevalence of double vision was $25.6 \%$ within the recurrent visual hallucinations group compared to $12.2 \%$ in the non-recurrent visual hallucinations group [16]. Racette et al. presented a patient with PD who developed horizontal diplopia during 'off' periods. Diplopia occurred nine years after the patient's first motor symptoms and in the neuro-ophthalmological work-up, he was diagnosed with convergence insufficiency, present only during the parkinsonian 'off' phase [15].

The most common causes of diplopia were convergence insufficiency, followed by a reduced fusion range, heterotropia, impaired conjugate eye movements, and impaired stereopsis $[12,13,15]$. Schindlbeck et al. [13] found that convergence insufficiency occurred in 55.6\% of PD patients with diplopia (compared to $11.1 \%$ without diplopia), and decompensated heterophoria occurred in $37.0 \%$ of patients with diplopia (compared to $6.7 \%$ without diplopia). Additionally, studies have found that the following visual disturbances are significantly more common among diplopia PD patients during neuro-ophthalmological examination: problems with spatial perception, blurred vision, problems with contrast sensitivity, intermittent divergent strabismus and weakness of the oculomotor muscles [12-14]. These visual disturbances occurred in $84 \%(\mathrm{n}=21)$ of PD patients with diplopia in Visser et al's [12] study. Additionally, visual hallucinations were also significantly more common in PD patients with diplopia [12, 13].

Based on the published studies, the following risk factors are considered to contribute to the development of diplopia (1) older age; (2) disease duration; (3) disease severity; (4) cognitive decline; (5) presence of visual hallucinations; and
(6) higher LED (594 mg for selective diplopia vs 1,075 mg for complete diplopia).

However, based on a logistic regression model with single predictors (e.g., presence of diplopia, presence of blurred vision, advanced disease duration and high LED), Schindlbeck et al. [11] concluded that the presence of diplopia was the only significant predictive factor for the occurrence of visual hallucinations, whereas disease duration, higher LED, and blurred vision had no significant influence on the presence of diplopia. Nebe et al. [14] found a correlation between the occurrence of diplopia and exposure to amantadine as well as up-titration of dopamine agonists or levodopa. Additionally, one patient in their study experienced diplopia after deep brain stimulation (DBS) [14].

\section{Discussion}

The prevalence of diplopia in $\mathrm{PD}$ patients ranges from 10\% to $30 \%[3,17]$. Diplopia is usually limited to specific situations such as reading or when looking around; however, it may also be constant. It is important to differentiate between monocular diplopia (double vision in only one eye, usually caused by intraocular pathology), binocular diplopia (double vision in both eyes, caused by ocular misalignment) and selective diplopia (diplopia of single objects) as a manifestation of visual hallucination or dementia in $\operatorname{PD}[3,18,19]$.

Binocular diplopia in PD can be related to convergence insufficiency or decompensated latent strabismus (e.g., deviation of the visual axis). Additionally, diplopia in PD can also be considered part of an 'off' phenomenon corresponding with motor fluctuations [3, 15, 18]. Patients with pre-existing ocular misalignment are also at risk of developing diplopia in PD. 


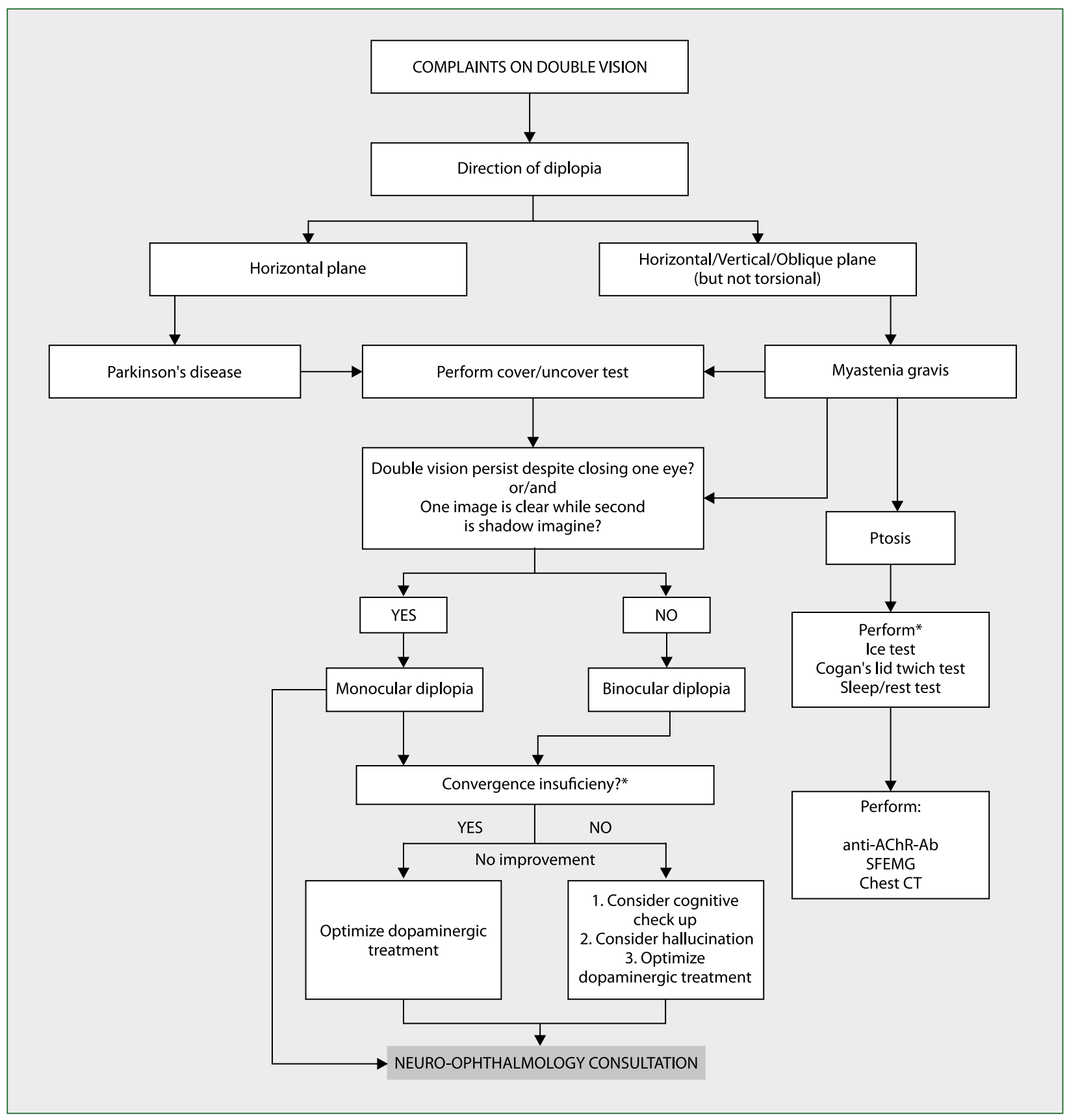

Figure 1. Flow chart with approach to double vision in Parkinson's disease (author's version). NMS - non-motor symptoms; anti-AChR-Ab anti-acetylcholine receptor antibodies; SFEMG - single-fiber electromyography; CT - computer tomography; * The physician holds a small target (e.g. pensil) in front of the patient and slowly moves it closer until either the patient have double vision or the physician sees an eye drift outward

The cover/uncover test can be performed as a screening tool to diagnose diplopia (Fig. 1). This test should be performed separately for both eyes (with one eye covered). Double images will disappear in binocular diplopia but persist in monocular diplopia [19]. Different forms of diplopia have been associated with both peripheral and central pathways. However, convergence insufficiency and heterophoria are significantly more frequent among PD patients with diplopia. As such, complaints of visual blurring, difficulties while reading and diplopia are common in PD. These complaints often raise questions as to whether they are related to
PD or rather reflect a coincidence with primary ophthalmological disturbances (which are common in the elderly population) or other co-morbidities (e.g., MG or vascular disease).

In this regard, we have included two case reports to underline two different causes of diplopia that clinicians may encounter when providing care for PD patients. It is therefore important to question the patient about which activities provoke diplopia and to differentiate from monocular diplopia (usually caused by suggested ocular media opacities such as a cataract or refractive problems). 
MG should therefore always be considered as a differential diagnosis of diplopia in PD.

Moreover, diplopia related to convergence insufficiency tends to improve with optimisation of dopaminergic therapy, suggesting basal ganglia involvement in its pathophysiology [20]. Additionally, Urwyler et al. [16] suggested extranigral pathology may be involved into diplopia due to convergence insufficiency. In summary, dopaminergic mechanisms, motor fluctuations, visual hallucinations and cognitive impairment should be considered, as these factors may also contribute to this heterogeneous symptom (e.g., selective diplopia).

The association between ocular symptoms and PD is not completely clear. Additionally, the most commonly used rating scale for PD, the Movement Disorders Society Unified Parkinson's Disease Rating Scale (MDS-UPDRS), does not include any questions regarding diplopia [21]. In the included studies, none of the patients mentioned their type of diplopia. As such, it is important for clinicians to complement the patient interview with a neuro-ophthalmological screening and to refer the patients to an ophthalmologist, if needed. This approach will prevent delayed diagnosis and further deterioration of clinical symptoms and quality of life.

Additionally, clinicians should be encouraged to adjust dopaminergic treatment while ocular symptoms occur. Interestingly, diplopia can also be a result of DBS or pallidotomy. Of note, Bejjani et al. [22] reported reversible acute diplopia with dystonic posture and tremor in four of 25 patients who underwent DBS when active electrodes were localized close to the red nucleus.

While diplopia can occur outside of $\mathrm{PD}$, there is evidence suggesting the co-occurrence of PD and MG. Sciacca et al. [23] reported three patients diagnosed with PD who developed MG. The first patient presented with bilateral ptosis and asthenia; the second patient developed bilateral ptosis and a dropped head; and the third presented with ptosis, diplopia and asthenia for 2 years. Only the third patient had positive titres for anti-acetylcholine receptor antibodies. All patients had typical changes in single-fibre electromyography, and all showed clinical improvement after the introduction of pyridostigmine.

Both PD and MG share several common features, including fatigue, diplopia, dysphagia and dysarthria, which may lead to misdiagnosis. The mechanism of the co-occurrence of $\mathrm{PD}$ and MG remains unclear, though there are two possible explanations. The first is iatrogenic; trihexyphenidyl can interfere with neuromuscular transmission by competing with the acetylcholine receptor $[24,25]$. The second is immune based on a reported association of $\mathrm{PD}$ with thyroid disease, $\mathrm{MG}$ and rheumatoid arthritis [26, 27].

The onset of diplopia in MG is usually gradual, with mild or intermittent symptoms. Diplopia in MG is usually accompanied by unilateral or bilateral ptosis along with other neurological signs (dyspnoea, dysphagia, dysphonia and weakness of the arm and leg muscles). There are some clinical features that can help to distinguish diplopia resulting from
PD vs MG. Diplopia in PD typically occurs in the horizontal plane, especially when the patient is tired, whereas diplopia in MG is more variable in direction (sometimes vertical and sometimes horizontal) and severity (from day to day and with diurnal fluctuations that worsen in the evening) [28].

To conclude, patients with PD can experience diplopia during the course of their disease. This has a clear impact on their health-related quality of life and safety (e.g., risk of falls). The acute onset of diplopia should always lead to tests to exclude MG and vascular disease. Tailored interventions should be developed to improve patient safety, quality of life and quality of care.

Ethical permission: Ethical approval was not necessary for the preparation of this article.

Funding: This publication was prepared without any external source of funding.

Conflicts of interest: The authors declare no conflict of interest.

\section{References}

1. Parkinson J. AN ESSAY ON THE SHAKING PALSY. Archives of Neurology. 1969; 20(4): 441-445, doi: 10.1001/archneur.1969.00480100117017.

2. Mueller C, Rajkumar AP, Wan YiM, et al. UK Parkinson's Pain Study Collaboration, EUROPAR and MDS Non-motor PD Study Group, National Institute for Clinical Excellence. Non-motor symptoms of Parkinson's disease: diagnosis and management. Lancet Neurol. 2006; 5(3): 235-245, doi: 10.1016/S1474-4422(06)70373-8, indexed in Pubmed: 16488379.

3. Ekker MS, Janssen S, Seppi K, et al. Ocular and visual disorders in Parkinson's disease: Common but frequently overlooked. Parkinsonism Relat Disord. 2017; 40: 1-10, doi: 10.1016/j.parkreldis.2017.02.014, indexed in Pubmed: 28284903.

4. Archibald NK, Clarke MP, Mosimann UP, et al. The retina in Parkinson's disease. Brain. 2009; 132(Pt 5): 1128-1145, doi: 10.1093/brain/ awp068, indexed in Pubmed: 19336464.

5. Nguyen-Legros J. Functional neuroarchitecture of the retina: hypothesis on the dysfunction of retinal dopaminergic circuitry in Parkinson's disease. Surg Radiol Anat. 1988; 10(2): 137-144, doi: 10.1007/ BF02307822, indexed in Pubmed: 3135618.

6. Davidsdottir S, Cronin-Golomb A, Lee A. Visual and spatial symptoms in Parkinson's disease. Vision Res. 2005; 45(10): 1285-1296, doi: 10.1016/j.visres.2004.11.006, indexed in Pubmed: 15733961.

7. Djamgoz MB, Hankins MW, Hirano J, et al. Neurobiology of retinal dopamine in relation to degenerative states of the tissue. Vision Res. 1997; 37(24): 3509-3529, doi: 10.1016/S0042-6989(97)00129-6, indexed in Pubmed: 9425527.

8. Clark D, Eggenberger E. Neuro-ophthalmology of movement disorders. Curr Opin Ophthalmol. 2012; 23(6): 491-496, doi: 10.1097/ ICU.0b013e328358ba14, indexed in Pubmed: 23014265.

9. Yoon WT. Comparison of dystonia between Parkinson's disease and atypical parkinsonism: The clinical usefulness of dystonia distribution and characteristics in the differential diagnosis of parkinsonism. Neurol Neurochir Pol. 2018; 52(1): 48-53, doi: 10.1016/j. pjnns.2017.11.004, indexed in Pubmed: 29196058. 
10. Alster P, Krzyżanowska E, Koziorowski $D$, et al. Difficulties in the diagnosis of four repeats (4R) tauopathic parkinsonian syndromes. Neurol Neurochir Pol. 2018; 52(4): 459-464, doi: 10.1016/j. pjnns.2018.06.002, indexed in Pubmed: 30025721.

11. Weil RS, Schrag AE, Warren JD, et al. Visual dysfunction in Parkinson's disease. Brain. 2016; 139(11): 2827-2843, doi: 10.1093/brain/ aww175, indexed in Pubmed: 27412389.

12. Visser F, Vlaar AMM, Borm CD, et al. Diplopia in Parkinson's disease: visual illusion or oculomotor impairment? J Neurol. 2019; 266(10): 2457-2464, doi: 10.1007/s00415-019-09430-w, indexed in Pubmed: 31214767.

13. Schindlbeck KA, Schönfeld S, Naumann W, et al. Characterization of diplopia in non-demented patients with Parkinson's disease. Parkinsonism Relat Disord. 2017; 45: 1-6, doi: 10.1016/j.parkreldis.2017.09.024, indexed in Pubmed: 28993094.

14. Nebe A, Ebersbach G. Selective diplopia in Parkinson's disease: a special subtype of visual hallucination? Mov Disord. 2007; 22(8): 11751178, doi: 10.1002/mds.21298, indexed in Pubmed: 17230466.

15. Racette BA, Gokden MS, Tychsen LS, et al. Convergence insufficiency in idiopathic Parkinson's disease responsive to levodopa. Strabismus. 1999; 7(3): 169-174, doi: 10.1076/stra.7.3.169.636, indexed in Pubmed: 10520242.

16. Urwyler P, Nef T, Killen A, et al. Visual complaints and visual hallucinations in Parkinson's disease. Parkinsonism Relat Disord. 2014; 20(3): 318-322, doi: 10.1016/j.parkreldis.2013.12.009, indexed in Pubmed: 24405755.

17. Savitt J, Aouchiche R. Management of Visual Dysfunction in Patients with Parkinson's Disease. J Parkinsons Dis. 2020; 10(s1): S49-S56, doi: 10.3233/JPD-202103, indexed in Pubmed: 32741840.

18. Borm CD, Smilowska K, de Vries NM, et al. How I do it: The Neuro-Ophthalmological Assessment in Parkinson's Disease. J Parkinsons Dis. 2019; 9(2): 427-435, doi: 10.3233/JPD-181523, indexed in Pubmed: 30958314.

19. Almer Z, Klein KS, Marsh L, et al. Ocular motor and sensory function in Parkinson's disease. Ophthalmology. 2012; 119(1): 178-182, doi: 10.1016/j.ophtha.2011.06.040, indexed in Pubmed: 21959370.
20. Lepore F. Parkinson's Disease and Diplopia. Neuro-Ophthalmology. 2009; 30(2-3): 37-40, doi: 10.1080/01658100600742838.

21. Siuda J, Boczarska-Jedynak M, Budrewicz S, et al. Validation of the Polish version of the Movement Disorder Society-Unified Parkinson's Disease Rating Scale (MDS-UPDRS). Neurol Neurochir Pol. 2020 [Epub ahead of print], doi: 10.5603/PJNNS.a2020.0049, indexed in Pubmed: 32639019.

22. Bejjani BP, Arnulf I, Houeto JL, et al. Concurrent excitatory and inhibitory effects of high frequency stimulation: an oculomotor study. J Neurol Neurosurg Psychiatry. 2002; 72(4): 517-522, doi: 10.1136/ jnnp.72.4.517, indexed in Pubmed: 11909914.

23. Sciacca G, Nicoletti A, Mostile G, et al. Is it just a coincidence? Three new cases of Myasthenia Gravis associated with Parkinson's disease. Parkinsonism Relat Disord. 2016; 28: 166-168, doi: 10.1016/j.parkreldis.2016.04.005, indexed in Pubmed: 27079256.

24. Iwasaki Y, Wakata N, Kinoshita M. Parkinsonism induced by pyridostigmine. Acta Neurologica Scandinavica. 1988; 78(3): 236-236, doi: 10.1111/j.1600-0404.1988.tb03653.x.

25. Levin N, Karussis D, Abramsky O. Parkinson's disease associated with myasthenia gravis. A report of 4 cases. J Neurol. 2003; 250(6): 766-767, doi: 10.1007/s00415-003-1088-y, indexed in Pubmed: 12862037.

26. Zis P, Argiriadou V, Temperikidis PP, et al. Parkinson's disease associated with myasthenia gravis and rheumatoid arthritis. Neurol Sci. 2014; 35(5): 797-799, doi: 10.1007/s10072-014-1660-5, indexed in Pubmed: 24500521.

27. Chaudhuri KR, Martinez-Martin P, Schapira AHV, et al. International multicenter pilot study of the first comprehensive self-completed nonmotor symptoms questionnaire for Parkinson's disease: the NMSQuest study. Mov Disord. 2006; 21(7): 916-923, doi: 10.1002/ mds.20844, indexed in Pubmed: 16547944.

28. Ueno S, Takahashi M, Kajiyama K, et al. Parkinson's disease and myasthenia gravis: adverse effect of trihexyphenidyl on neuromuscular transmission. Neurology. 1987; 37(5): 832-833, doi: 10.1212/ wnl.37.5.832, indexed in Pubmed: 3033545. 